Accepted by The Astrophysical Journal on 2019 September 30

HTTPS://DOI.ORG/10.3847/1538-4357/AB4A0A

Preprint typeset using $\mathrm{L}_{\mathrm{A}}^{\mathrm{T}} \mathrm{E}$ X style emulateapj v. 12/16/11

\title{
MULTIWAVELENGTH STEREOSCOPIC OBSERVATION OF THE MAY 1, 2013 SOLAR FLARE AND CME
}

ERICA Lastufka

Fachhochshule Nordwestschweiz,

Bahnhofstrasse 6, 5210 Windisch, Switzerland; and ETH-Zürich,

Rämistrasse 101, 8092 Zürich, Switzerland;

SÄM KRUCKER

Fachhochshule Nordwestschweiz,

Bahnhofstrasse 6, 5210 Windisch, Switzerland; and

Space Science Laboratory, UC Berkeley

Berkeley, USA;

IVAN Zimovets

Russian Academy of Sciences (IKI), 84/32 Profsoyuznaya Str, Moscow, Russia, 117997

izamov

Lomonosov Moscow State University,

Universitetskii Pr. 13, Moscow, Russia, 119992

Stephen White

Air Force Research Laboratory, Albuquerque, NM, USA

Satoshi Masuda

Nagoya University, Nagoya 464-8601, Japan

DMitriy Golovin

Russian Academy of Sciences (IKI),

84/32 Profsoyuznaya Str, Moscow, Russia, 117997

Maxim LitvaK

Russian Academy of Sciences (IKI),

84/32 Profsoyuznaya Str, Moscow, Russia, 117997

IGOR MitrofANOV

Russian Academy of Sciences (IKI),

84/32 Profsoyuznaya Str, Moscow, Russia, 117997

AnTon SANin

Russian Academy of Sciences (IKI),

84/32 Profsoyuznaya Str, Moscow, Russia, 117997

Accepted by The Astrophysical Journal on 2019 September 30

https://doi.org/10.3847/1538-4357/ab4a0a

\section{ABSTRACT}

A M-class behind-the-limb solar flare on 1 May 2013 (SOL2013-05-01T02:32), accompanied by a ( $400 \mathrm{~km} / \mathrm{s}$ ) CME was observed by several space-based observatories with different viewing angles. We investigated the RHESSI-observed occulted hard X-ray emissions that originated at least $0.1 R_{\mathrm{S}}$ above the flare site. Emissions below $\sim 10 \mathrm{keV}$ revealed a hot, extended (11 MK, >60 arcsec) thermal source from the escaping CME core, with densities around $10^{9} \mathrm{~cm}^{-3}$. In such a tenuous hot plasma, ionization times scales are several minutes, consistent with the non-detection of the hot CME core in SDO/AIA's $131 \AA$ filter. The non-thermal RHESSI source originated from an even larger area ( 100 arcsec) at lower densities $\left(10^{8} \mathrm{~cm}^{-3}\right)$ located above the hot core, but still behind the CME front. This indicates that the observed part of the non-thermal electrons are not responsible for heating the 
CME core. Possibly the hot core was heated by non-thermal electrons before it became visible from Earth, meaning that the un-occulted part of the non-thermal emission likely originates from a more tenuous part of the CME core, where non-thermal electrons survive long enough to became visible from Earth. Simultaneous hard X-ray spectra from the Mars Odyssey mission, which viewed the flare on disk, indicated that the number of non-thermal electrons $>20 \mathrm{keV}$ within the high coronal source is $\sim 0.1-0.5 \%$ compared to the number within the chromospheric flare ribbons. The detection of high coronal hard X-ray sources in this moderate size event suggests that such sources are likely a common feature within solar eruptive events.

Keywords:

\section{INTRODUCTION}

Coronal hard X-ray (HXR) sources provide one of the most exciting diagnostics of coronal plasma. However, the majority of flare-associated HXRs that we can observe occur at the footpoints of flare loops, when accelerated electrons collide with dense chromospheric plasma. Because these sources are orders of magnitude brighter than coronal HXR emission, most X-ray instruments are incapable of resolving both coronal and chromospheric sources simultaneously.

Coronal X-ray emission can have both thermal and non-thermal components. These can be difficult to distinguish from each other in the low corona, where accelerated electrons interact with dense arcades of cooling flare loops. For less powerful or more compact flares, looptop sources, or indeed any X-ray source located even higher up in the corona (hard X-ray sources have been observed up to $0.3 R_{\odot}$ ), are only observable when the flare footpoints are occulted behind the solar disk. Only in certain spectacular cases, such as the Masuda flare (Masuda et al. 1994), can the thermal and non-thermal components be well-separated spatially by dynamic-range limited intsruments, without the additional advantage of footpoint occultation.

Observations of both occulted flares and Masuda-type events have had profound impacts on our understanding of particle acceleration in flares. Mechanisms of particle acceleration, trapping, or turbulence that are responsible for producing bremsstrahlung in what is traditionally thought of as tenuous coronal plasma are not yet well understood. Multi-wavelength observations of coronal emission, as well as of the complete flare, are the keys to characterizing particle acceleration from one region to the next.

Unfortunately, comprehensive observations of occulted flares, especially those with X-ray sources very high in the corona, are rare. Two of the best-observed examples are the X-class flare of October 27, 2002 Krucker et al. 2007) and the smaller ${ }^{1}$ flare of November 3, 2010 (Glesener et al. 2013). The powerful October 27 flare showed an extended coronal source that moved rapidly $(750 \mathrm{~km} / \mathrm{s})$ in the same direction as the accompanying coronal mass ejection (CME). An estimated 10\% of the electrons in the source were determined to be nonthermal, possibly particles trapped on field lines related to the CME.

Another facet of one global magnetic eruption, CMEs, which often accompany flares (Harrison 1995. Zhang et al. 2001), can contribute to the presence of locally dense plasma capable of producing enhanced

\footnotetext{
${ }^{1}$ The unocculted SXR flux registered as GOES C5 class. Chertok's method gave a class of X1.5.
}

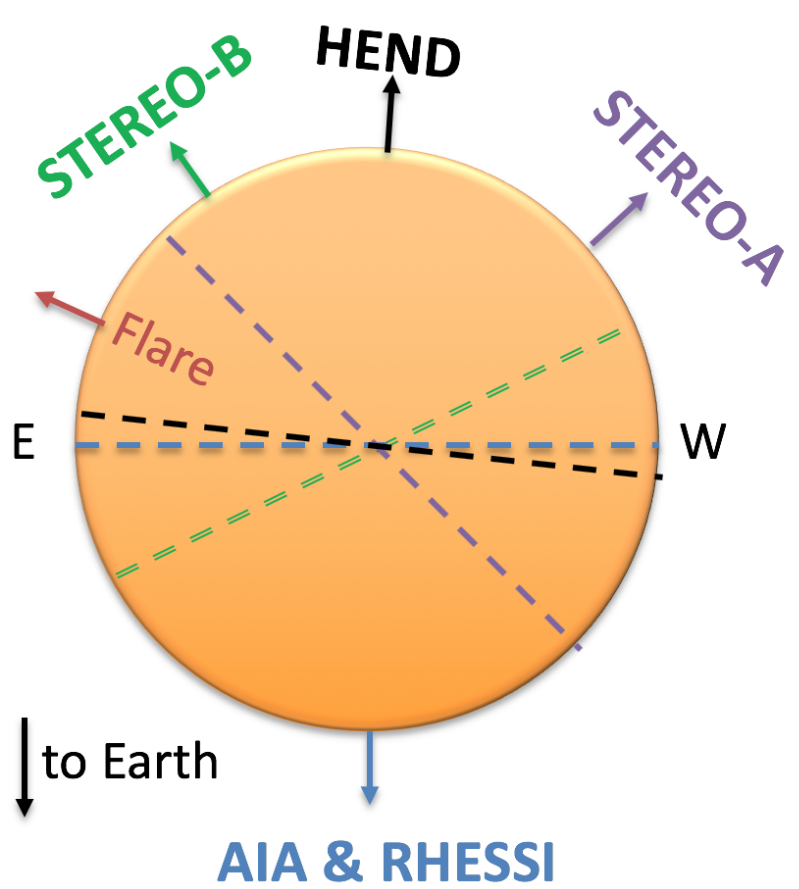

Figure 1. The flare of May 1, 2013, was observed from many perspectives. This diagram imagines looking down on the ecliptic; solid vectors point towards the satellites, and dashed lines represent the half of the Sun visible from each perspective.

bremsstrahlung. As a flux rope rises from the solar surface, a current sheet forms below it, containing microscopic instabilities resulting in fast magnetic reconnection. Following reconnection, the envelope of field lines overlying the flux rope is pushed up, forming the CME's bright frontal loop with a piston-driven shock ahead of it. Part of the flux rope is now known as the CME core, while the remainder falls to the solar surface (Gopalswamy et al., 2003; Jing et al., 2004). High-energy particles and hot plasma can be released in bi-directional outflows observable in radio and X-rays (Liu et al. 2008). Their motion can often be traced by co-spatial soft Xray (SXR) or EUV plasmoids that form as the upward reconnection flow hits the CME core.

Hard X-rays have also been observed moving with CME cores (Hudson et al. 2001). Flare-accelerated electrons in the CME core can cause the thermal CME energy to exceed the CME kinetic energy (Lee et al. 2009. Landi et al. 2010), resulting in nonthermal electron energy loss, observed through HXR emission. The November 3 flare studied by Glesener et al. (2013) is a prime example - simultaneous EUV and X-ray observations showed that flare-accelerated electrons had enough energy to heat the CME core. It is clear that the dynamics 

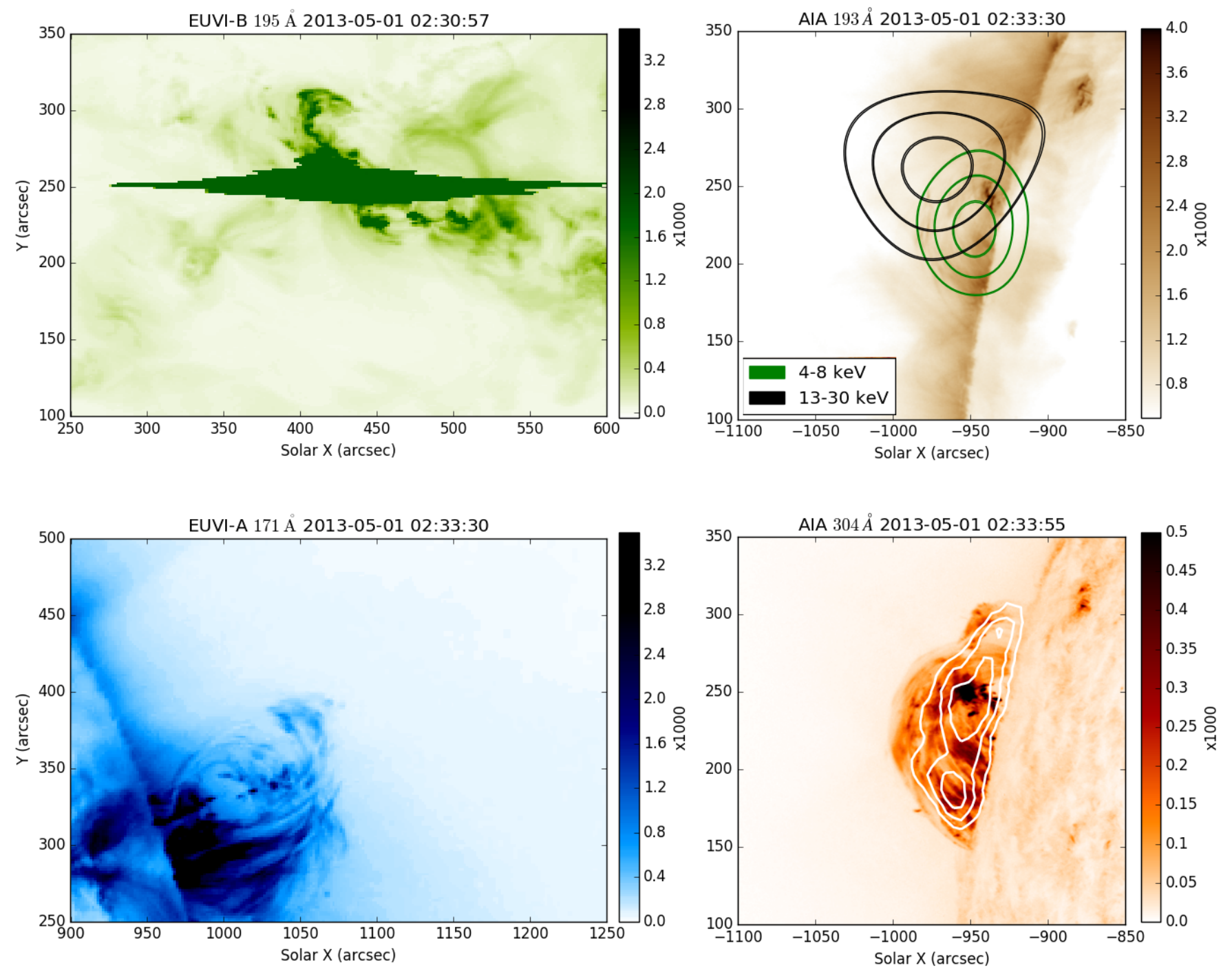

Figure 2. From top-left, clockwise: The flare peak observed directly by STEREO-B $195 \AA$. RHESSI imaging of the flare peak at 02:33UT showed an extended non-thermal source leading a compact thermal source. No corresponding sources were visible in AIA $193 \AA$. However, plasma observed at $17 \mathrm{GHz}$ by NoRH resembled the cooler-temperature AIA $304 \AA$ images. The view from STEREO-A was occulted by $3^{\circ}$ less than AIA, so it saw slightly more of the flare. An animated version of this figure is available, following the flare evolution. From an hour before the flare erupts, the full-disk view offered by STEREO-B shows the active region changing. During and after the flare peak, short-lived X-ray emission and longer-lived radio emission is seen. The filament eruption is seen especially well from the STEREO-A viewpoint, with EUV plasmoids stretched along the filament axis seven minutes after the peak.

of CMEs and the energy release of flares are intrinsically coupled to each other.

The event of May 1, 2013 (SOL2013-05-01T02:32) is an excellent opportunity to investigate how and where a solar eruption accelerates particles. Observed by an array of instruments ranging in wavelength from radio to hard X-ray, it enables the study of the coronal electron population at many temperatures. In this paper, we focus on both the potential causes of the observed X-ray emission and its properties.

\section{EVENT DESCRIPTION}

\subsection{Observation Geometry}

The active region that produced the May 1, 2013 flare was fully visible to STEREO-B's SECCHI (Howard et al. 2008), to the north-east of its field of view. The centroid of the peak flare image gave a precise location of $\left(424^{\prime \prime}, 241^{\prime \prime}\right)$, which allowed us to infer the observation geometry. The flare position was located $30^{\circ}$ behind the east solar limb as seen from Earth, which meant that the observed plasma was emitted from heights at least 111 $\mathrm{Mm}$ radially above the active region. Mars Odyssey saw almost the opposite view from that of AIA, with the flare seen close to the limb but on disk. STEREO-A saw the flare as occulted by $27^{\circ}$ behind STEREO-A's east limb, which allowed plasma $88 \mathrm{Mm}$ or more above the flaring region to be observed. Figure 1 shows the approximate location of the flare with respect to the various observatories, and their fields-of-view at the time.

\subsection{Event Overview}

Figure 2 shows views from various instruments near the peak of the flare (02:32:28UT in RHESSI 13-30 keV), and the accompanying animation shows the four-hour period 
surrounding the event for context. Lightcurves, shown in Figure 3 for selected instruments are shown over that same time period (left panels) or in detail when the HXR emission occurs (right panels).

EUV views from the occulted perspectives - AIA and STEREO-A - showed varying amounts of increase in the hour leading up to the flare. The direct view of STEREO-B showed loops brightening and changing over the active region as it evolved. This behaviour is consistent with the appearance of loops over the solar limb as seen in the movie, and was accompanied by an increase in soft X-ray flux as seen by GOES SXI. After the flare peaks, dimming was seen in many EUV wavelengths, including material seen in absorption by STEREO-B. Shortly after the flare peak, STEREO-A presented a beautiful view of an erupting filament in both its channels, with small, bright plasmoids running up the axis of the filament. This was less spectacularly seen by AIA's EUV channels, perhaps due to the higher occultation. Post-flare loops were slow to emerge, with the first candidates being seen at 03:00UT by STEREO-A. Likely the high occultation angle, combined with an unfavorable loop orientation, allowed only the tops of the highest cooling loops to be seen from the occulted perspective.

In the X-ray regime, flux initially decreased as a previous flare on the solar disk decayed. However, after 2:20UT, RHESSI detected a second fainter source above the limb that would peak little over ten minutes later. The RHESSI 10-30 keV peak occurred at 02:32:28UT and the 4-8 keV peak was slightly delayed at 02:34:15UT (see right panel, Figure 3). The High Energy Neutron Detector (HEND), part of the Gamma Ray Spectrometer aboard Mars Odyssey, saw the main peak at 02:32:09UT in channels from $65-200 \mathrm{keV}$. It observed several bursty peaks starting five minutes before the main peak $^{2}$. GOES XRS did not register this emission as a flare. At the time of the RHESSI HXR peak, the amount of flux in the $1-8 \AA$ channel corresponded to a $\mathrm{C} 1$ background with a B1 enhancement.

Because the majority of the event was occulted as seen from Earth, we used the STEREO-B EUV images to better estimate the flare magnitude. Following the method of Chertok et al. (2015), the 286" length of the saturated portion of the peak STEREO-B image at 02:30:57UT gave a flare class of M7. The method of Nitta et al. (2013), which compares the full-disk EUV flux before and during the flare, gave a GOES class of M3. Both these empirically derived formulae have large uncertainties, with Chertok claiming an accuracy of less than a factor of 2 and Nitta within 0.5-1.5 times the actual SXR flux value. Because the STEREO peak image occurred almost three minutes before the peak SXR flux, it is safe to assume that the flare was at least a moderate M-class but not as large as X-class.

RHESSI imaging, which will be discussed in more detail in sections 4.1 and 4.2 showed both thermal and nonthermal sources. The thermal source was visible for 5.5 minutes, outlasting the non-thermal source which could only be imaged over three minutes. The non-thermal source appeared both higher in the corona and earlier

2 This flare was not listed in the HEND flare catalog Livshits et al. 2017) because the corresponding GOES flux increase was not enough to indicate a flare due to the occultation. than any emission shown by the NoRH 17 and $34 \mathrm{GHz}$ images, which corresponded well with the AIA $304 \AA$ images, showing a large expanding bubble which lasted long after the X-ray sources faded. Both X-ray sources were clearly located behind the CME front. The CME itself, as seen by AIA, had a multi-thermal structure whose 3D nature was unclear due to projection effects. The hotter FeXVIII image showed most material concentrated in a single loop towards the north, while cooler plasma was distributed much further south and higher above the limb.

\section{SPECTRAL ANALYSIS}

\subsection{RHESSI thermal spectra}

Spectral analysis of the RHESSI thermal emission was complicated by the simultaneous occurrence of a decaying microflare located on disk. This made it difficult to fit the total flare spectrum integrated over the entire Sun; cross-talk at low energies resulted in a poor fit of a single thermal component. In order to cleanly isolate the two widely separated sources, we applied standard RHESSI imaging spectroscopy (Krucker and Lin (2002)). Using the coarsest subcollimators to distinguish coronal from on-disk emission enabled us to more accurately determine the properties of the thermal component.

Fitting a single temperature model gave the temperature and emission measure (EM) evolution shown in Figure 4, with moderately high temperatures peaking around 11 MK. The EM time evolution was found to be very different from that of a standard flare. Instead of a fast decay following the flare peak, the emission measure, shown here in linear scale, had unusually small changes. This highlighted the fact that we were not observing the expected flare loops, but emission from well above the actual flare site. This was also influenced by the motion of the thermal source and the associated change in the degree of occultation. In any case, it is not clear how to explain the nearly constant EM.

We estimated the volume of X-ray emitting thermal plasma by forward fitting a circular source to the RHESSI visibilities of all detectors (e.g. Dennis and Pernak (2009)). This revealed an extended source with FWHM sizes above $\sim 60$ " for all times, and a clear trend of an increasing size after 02:33UT. Assuming a spherical symmetry and filling factor of unity, the derived density of the hot $(\sim 11 \mathrm{MK})$ plasma was around $10^{9} \mathrm{~cm}^{-3}$, a plausible density for this CME core in the high corona. The filling factor is an unknown; RHESSI observations, with a limited number of measured visibilities, cannot distinguish between an extended source and a composite of many subsources. Introducing a filling factor could drastically increase the density; however, as we will later discuss, a filling factor of unity agrees well with the data for this particular event.

\subsection{RHESSI \& HEND Hard X-ray Spectra}

This flare was one of very few where spectroscopic observations of both the chromospheric footpoints and the coronal source were available. Measurements of the footpoint emission came from the High Energy Neutron Detector (HEND), part of Mars Odyssey's Gamma Ray Spectrometer (GRS) instrument suite (Boynton et al. 2004 ), which observed energetic particles from $\approx 32-2000$ 

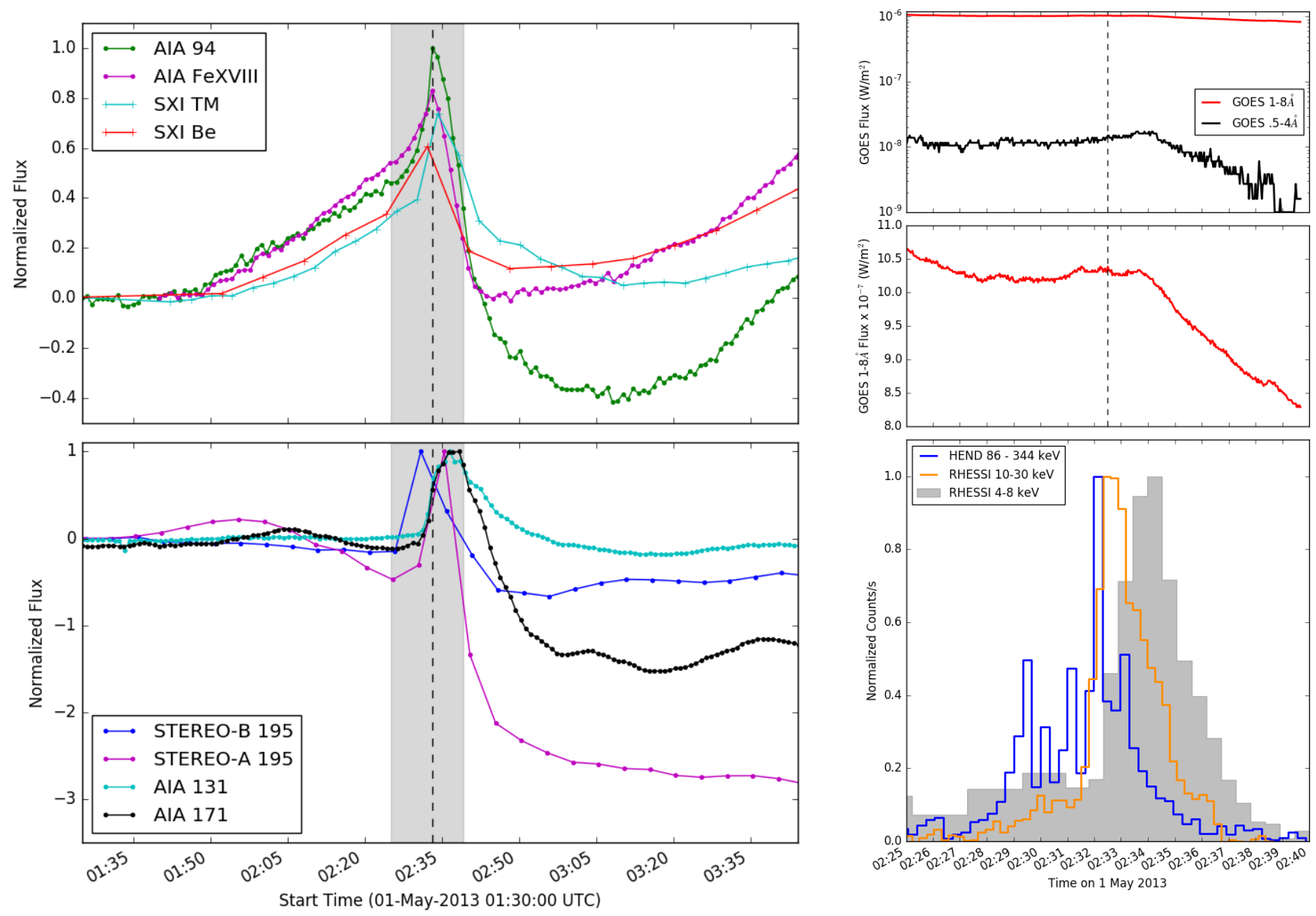

Figure 3. Left panels: Lightcurves from instruments sensitive to hot plasma (top) and cooler plasma (bottom), for the same time period shown in the animated version of Figure 2 Flux was determined from an area 400" x 400" either centered on the flare (direct perspective of STEREO-B) or above the limb but in the same vertical extent (all occulted perspectives). Each lightcurve was background-subtracted using pre-flare values and normalized relative to the flare peak. The symbols indicate the imaging time cadence, which influences the relative timing of the peaks, especially in the bottom panel. The shading indicates the time period illustrated in further detail on the right. Right panels: GOES, RHESSI, and HEND lightcurves. The GOES long channel, shown in linear scale (middle) shows an increase of flux corresponding to the RHESSI emission, overlaid on a decaying slope. The direct view of HEND observed bursts before the main flare onset.

$\mathrm{keV}$. At the time of observation, the spacecraft structure did not shade the detector, simplifying interpretation of the detected flare X-ray photons. The peak in the observed count spectrum was strongest in the $86-344 \mathrm{keV}$ range. Even though this was a M-class flare, there were more than enough counts to clearly both distinguish solar emission from the background up to $400 \mathrm{keV}$, and render the errors negligible.

Figure 3 shows that the RHESSI and HEND emission peaked almost simultaneously for the high-energy channels. During calibration, the HEND data was adjusted for the light travel time between Mars and Earth orbits. The HEND > $90 \mathrm{keV}$ peak, beginning at 02:32:09UT with a time binning of $\approx 20$ seconds, might well have overlapped the RHESSI $10-30 \mathrm{keV}$ peak in the 4-second rotation from 02:32:28-02:32:32UT. HEND showed multiple smaller peaks indicating high-energy bursts in the chromosphere starting at 02:28UT, before RHESSI registered the coronal source.

An OSPEX fit of the photon spectrum integrated over the main non-thermal peak (02:32:00-02:33:13UT) to a power law with a low-energy cutoff gave slopes around $\gamma_{c o r}=3.3 \pm 0.38$, where the uncertainties were derived from the standard deviations between the fit results of the individual RHESSI detectors. The flare-integrated spectra showed that non-thermal emission was the main component above $13 \mathrm{keV}$ and extended down to at least $10 \mathrm{keV}$, below which thermal emission dominated. A single power-law fit to the HEND spectrum, excepting the two highest energy bins which had significant uncertainty, gave a spectral index of $\gamma_{\text {chrom }}=2.47 \pm 0.26$. The difference between the spectral indices of the coronal source (RHESSI) and footpoints (HEND) is within the range observed by Battaglia and Benz (2006) for flares with distinct above-the-looptop coronal and footpoint Xray sources. Extrapolating the RHESSI fit to $100 \mathrm{keV}$, where the observed signal is difficult to distinguish from the background, we see that the photon flux observed by HEND was $\approx 10^{3}$ more than that observed by RHESSI.

\section{CORONAL SOURCE EVOLUTION}

\subsection{RHESSI imaging of the thermal emission}

We used RHESSI forward fitting to determine the size and position of the 4-8 keV X-ray source. Not only did 


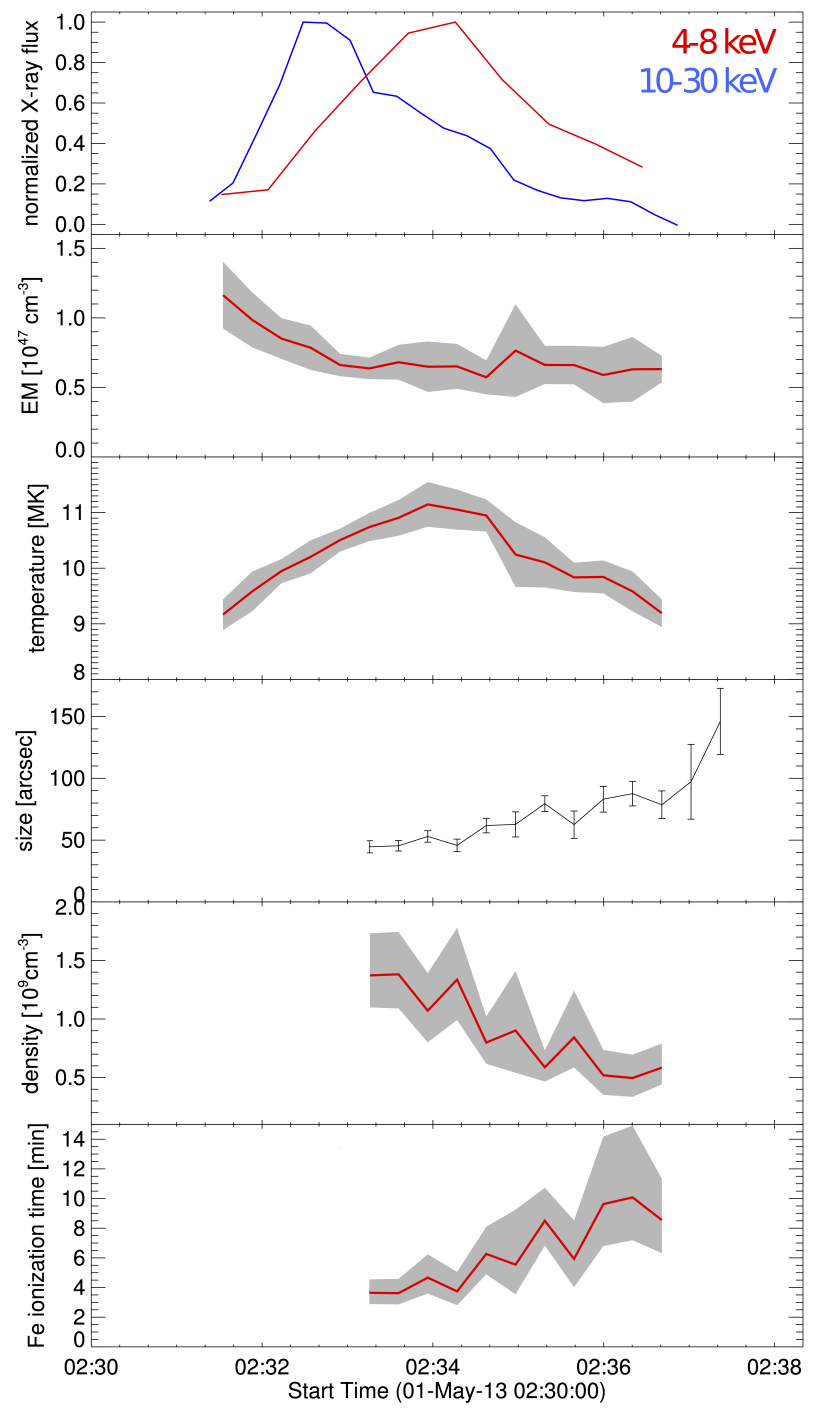

Figure 4. Properties derived from the RHESSI SXR source (red curve, top plot). The emission measure stayed surprisingly constant, although the temperature followed the expected behaviour. The last three panels, which depended on imaging, are shown when the flare fluxes clearly exceed the flux of the on-disk source. The compact thermal source almost doubled in size as it rose above the flaring region and the solar limb. The density, calculated using a filling factor of unity, follows the temperature profile. Long iron ionization times might explain why AIA did not observe the thermal source.

this allow us to separate the on-disk and coronal source, but also to determine the optimal parameters for imaging the thermal source with CLEAN. First, the source size and position was calculated by forward fitting. Next, images for each subcollimator separately were reconstructed using CLEAN. If the source position agreed with the position calculated by the forward fit, that subcollimator was selected for use in making the final CLEAN images shown in Figures 6 and 7

The CLEAN images show the expansion and movement of the compact thermal source. The imaging, when overlaid on AIA $304 \AA$, clearly showed that the thermal emission came from the core of the CME. This particular AIA filter sees cold plasma, which exhibited fine structure within the CME core. In this event, cold and hot

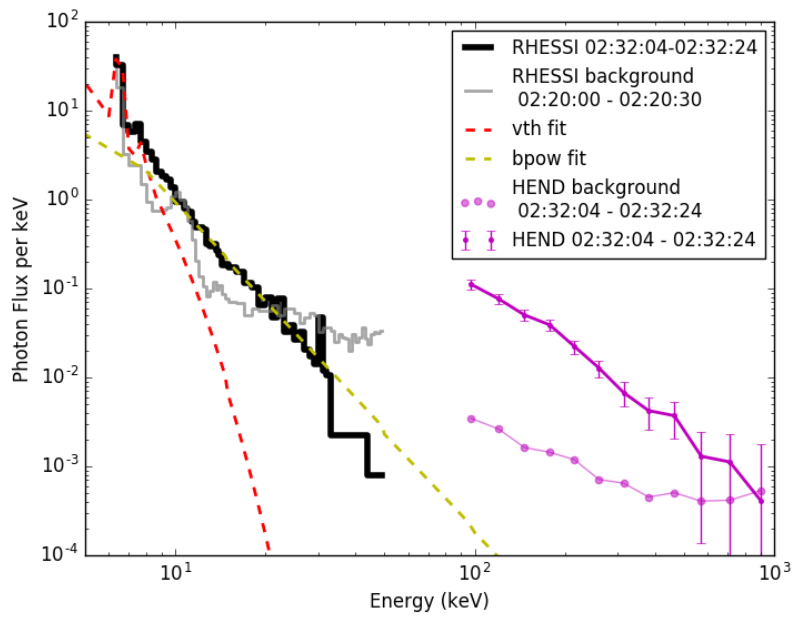

Figure 5. RHESSI and HEND photon spectra. The RHESSI photon spectrum was fit with both a single thermal component plus a broken power-law.

plasma must have co-existed in the core, at least in projection.

A fit to the $4-8 \mathrm{keV}$ centroid positions over time gave an average velocity of $150 \mathrm{~km} / \mathrm{s}$. This was much slower than the linear CME speed, measured by LASCO as $389 \mathrm{~km} / \mathrm{s}$. The source rose to a maximum height of $73 \mathrm{Mm}$ above the limb, or $184 \mathrm{Mm}$ (243") above the active region. This is one of the best RHESSI image sequences to date of a purely coronal source from within the core of a $\mathrm{CME}$ rising and expanding above the limb.

\subsection{RHESSI imaging of the non-thermal emission}

From the RHESSI spectral analysis (section 3.2), we concluded that emissions above $13 \mathrm{keV}$ were purely nonthermal. Above $30 \mathrm{keV}$, the background began to dominate the count spectrum. We therefore selected the energy range from $13-30 \mathrm{keV}$ for imaging the non-thermal source. Relative to the thermal source, count statistics were low, with only about 1000 counts per detector if integrated over the entire non-thermal peak, so to summarize we reconstructed a single image averaged over the duration of the non-thermal burst (02:32:0302:33:21UT). For reference we made an image of the thermal source in the further restricted 6-8 keV energy range integrated over the same time interval.

Using a forward fit, we found that the thermal source came from an extended $\left(61 \pm 10^{\prime \prime}\right.$ FWHM) area. The nonthermal emission came from an even larger (110 $\pm 30 "$ FWHM) area. The center of mass location difference for the thermal and non-thermal source of $41 \pm 13$ " in the $\mathrm{x}$ - and $39 \pm 10^{\prime \prime}$ in $\mathrm{y}$ - direction, or a radial separation of $56.6 \pm 16 "$. This clearly established that the nonthermal source was above the thermal CME core. For the summary image shown in Figure 7, we then used the CLEAN algorithm to make images without restricting the source shape, again allowing the source size derived from forward fitting to guide the subcollimator selection. We chose subcollimators 6 through 9 for the thermal source and subcollimators 7 through 9 for the non-thermal source.

Note that the limb in EUV is slightly higher $\left(\sim 10^{\prime \prime}\right)$ than the X-ray limb (e.g. Battaglia et al. (2017)), so the 

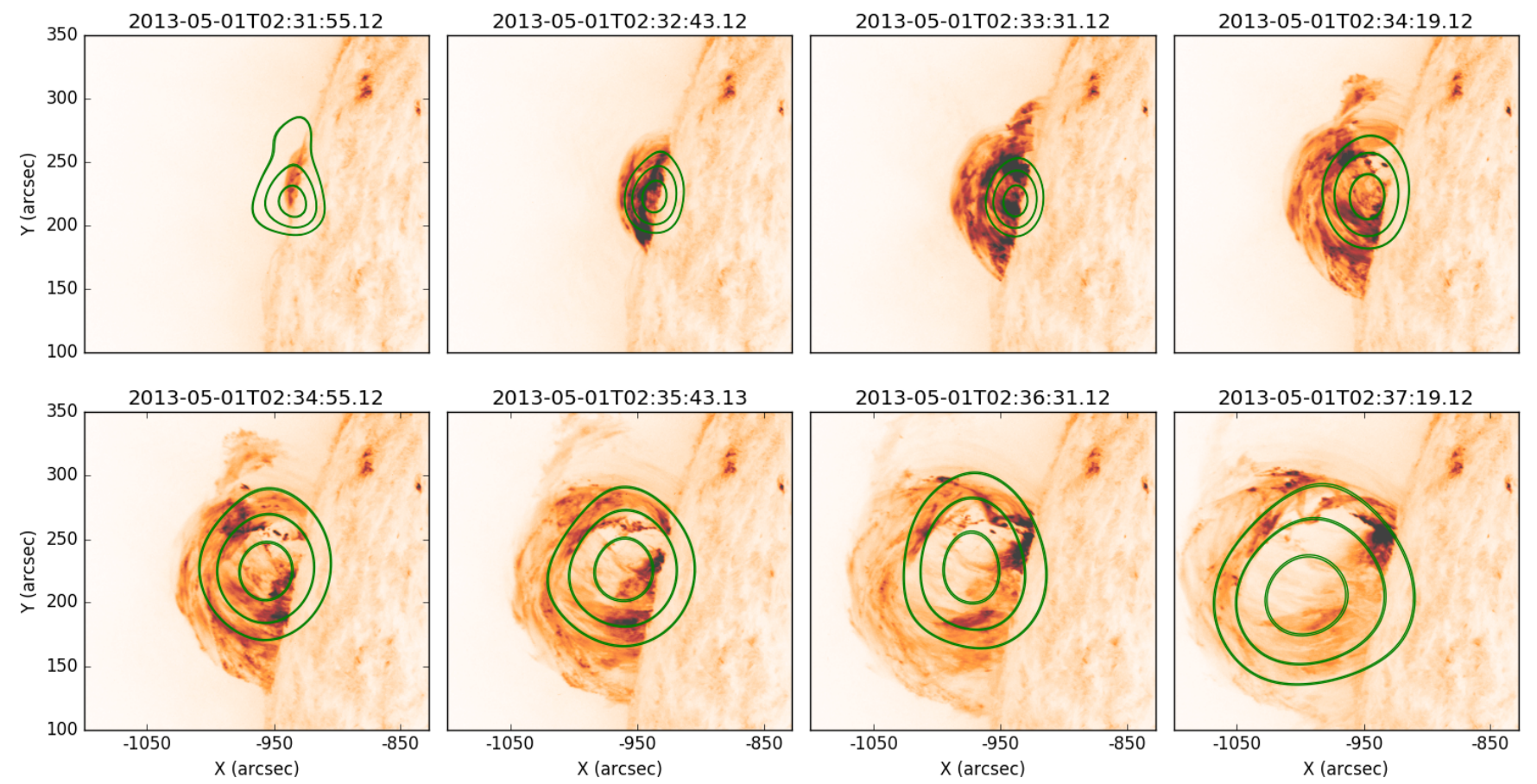

Figure 6. Time evolution of the soft 4-8 keV X-ray source. Contour levels are 50, 70 and 90\%, as is common with RHESSI imaging. SXR images, made every 45 seconds starting at 02:32UT, show that the compact source expanded as it moved upward above the flare region. The background images are from AIA $304 \AA$, corresponding to the timestamps.

$\mathrm{X}$-ray source appeared at slightly lower altitude. The non-thermal source was clearly outside the CME core and just ahead of it, but still much behind the CME front even with its multi-thermal structure.

\subsection{Nature of the non-thermal coronal emission}

We estimated the instantaneous number of electrons that are required to produce the observed hard X-ray emission using equation 2.4 from Lin (1974). Such an estimate depends on parameters of the observed hard $\mathrm{X}$-ray spectrum and the ambient density within the nonthermal source - that is to say, the thermal core distribution. While the absolute value and the slope of the power spectrum was well observed, the cutoff energy of the non-thermal spectrum and the ambient density are not well-constrained by the observations. Hence, the instantaneous number of non-thermal electrons and therefore the instantaneous number density can only be estimated for a range of parameters.

Figure 8 (top) gives the density of the instantaneous electrons as a function of ambient density for three different cutoff energies. The solid line represents the extreme case where the non-thermal density is equal to the ambient density. In this case, the derivation is no longer valid since collisions between non-thermal electrons should be considered as well. Generally, the non-thermal component is thought of as a tail on the thermal core distribution that only represents a small fraction of the total particles in the distribution. The ambient density within the non-thermal source is not well constrained, but due to its higher altitude it should be below or possibly as high as the density of the hot core $\left(10^{9} \mathrm{~cm}^{-3}\right.$, see Figure 4.).

An estimate of the ambient density within the non- thermal source can be approximated if we assume that non-thermal electrons are trapped within the source and the observed decay of the non-thermal emission is due to collisional losses only. In this case, the collisional stopping time (e.g. Krucker and Lin (2008), eq 2) should be roughly equal to the observed exponential decay time of 141 seconds $(13-30 \mathrm{keV})$. The bottom panel of Figure 8 shows the energy loss time as a function of density for different electron energies. This indicates that densities around $10^{8} \mathrm{~cm}^{-3}$ give plausible energy loss times.

An alternative theory is that the observed flux decay results from a rapidly decreasing ambient density as the bubble trapping the non-thermal electrons expands. In such a model, the thin target emission, which is proportional to the ambient density, decreases in time. Therefore the hard X-ray flux also decreases. The collisional losses also decrease with time, meaning that the non-thermal electrons could potentially survive for a long time within the escaping bubble. For a constant, isotropic expansion velocity, density decreases with time proportional to the third power. As the exponential fit mentioned previously is only observed over a short time interval, the decay can also be approximated with a power-law decay, at least for this event with limited counts. For isotropic expansion velocities of the order of $200 \mathrm{~km} / \mathrm{s}$, the observed decay can be roughly reproduced, assuming the injection stops at the peak time of the non-thermal emission and using the observed source size at that time of 110". As the CME is expanding with roughly double that speed (see Table 1), it is appears possible that the escaping bubble could move out at $200 \mathrm{~km} / \mathrm{s}$. For events with better statistics than for the May 1 event, the fit to the decay time could clearly distinguish between the two scenarios - collisional decay 

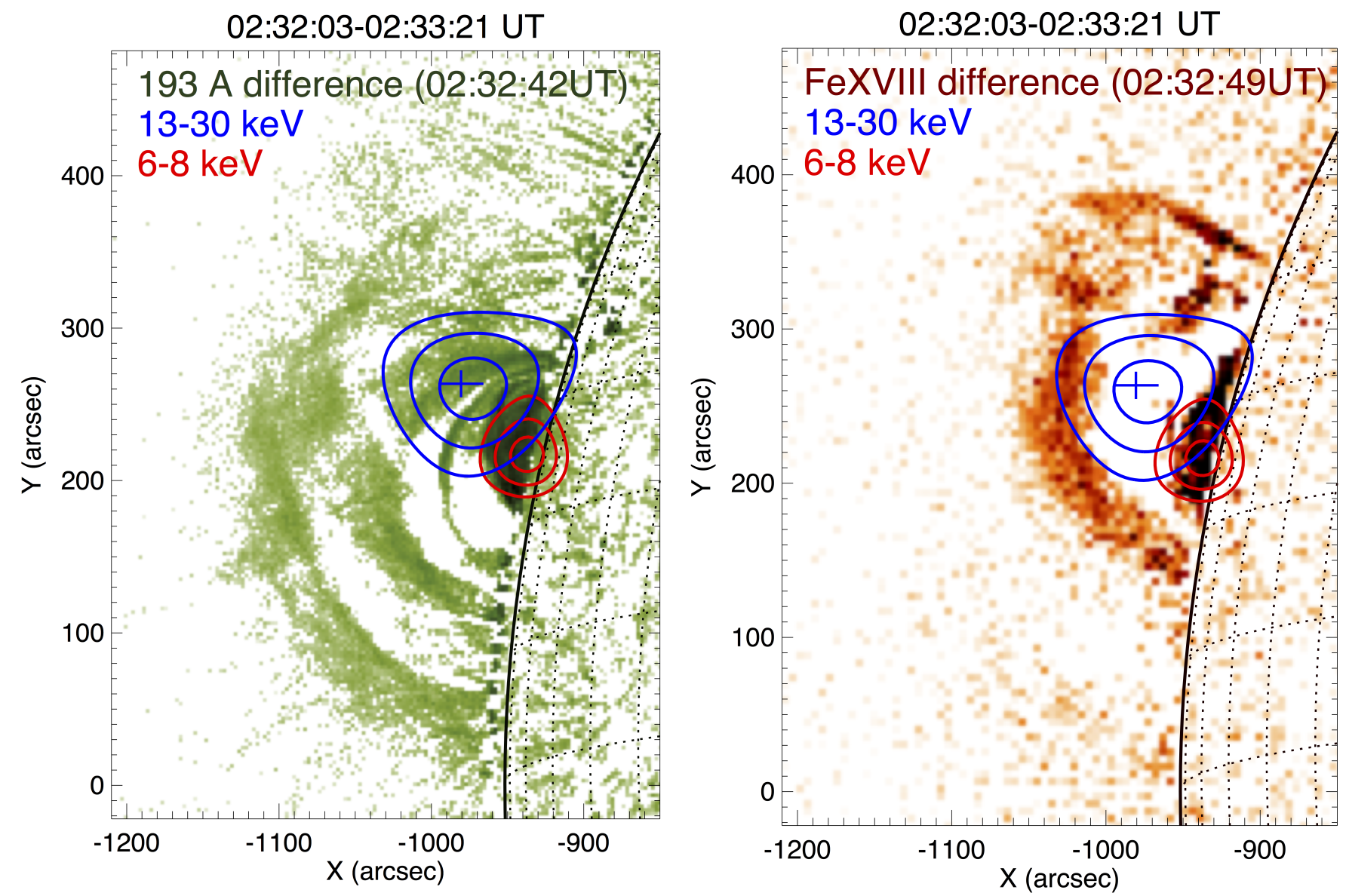

Figure 7. The RHESSI HXR source is best summarized by this image. Low counts meant that a multi-step process was needed to ensure an accurate source size and position. During the flare peak, the extended HXR source lead the compact SXR source, both of which were high above the flare loop arcade but behind the CME front. Note that the CME front, likely due to projection effects and the 3-D structure of the event, appears to be located more to the north in the hotter FeXVIII image than for the cooler 193 image.

vs expansion. For the October 27 flare (Krucker et al. 2007), which has 30 times higher count rates (see 'Table 5), an exponential decay is clearly preferred (Figure 1 and 3 of Krucker et al. (2007)). Nevertheless, as the bubble of non-thermal electrons is most likely expanding during the time scale of the collisional losses, the effect of decreasing ambient density should be considered in addition to collisional losses.

In summary, we get a consistent picture assuming that the non-thermal emission is produced by a trapped population of energetic electrons within a plasma with an ambient density of $\sim 10^{8} \mathrm{~cm}^{-3}$. For such densities, the fraction of the non-thermal population is reaching values on the order of a percent (c.f. Figure 8 top). Considering that the non-thermal electrons at $20 \mathrm{keV}$ have roughly 100 times more energy than an average electron in the ambient corona at $2 \mathrm{MK}$, the energy content of the non-thermal population could be similar to the ambient energy and therefore might play a significant role in the total evolution of the event.

\subsection{Coronal source at other wavelengths}

Although the pre-flare phase lightcurves showed flux increasing in AIA's hot channels, AIA images during the flare peak did not show a source in the corona corresponding to the thermal X-ray source. This is unlike the similar November 3 flare studied in Glesener et al. (2013), which clearly showed the $11 \mathrm{MK}$ CME core (the same temperature as derived for the CME core in this event).

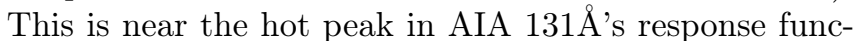
tion, due to FeXX and FeXXIII emissions. At this temperature ionization time scales for a low density plasma are not instantaneous. Ionization takes a few seconds or even minutes (e.g. Bradshaw and Raymond (2014)). Using the curves given in Smith and Hughes (2010) for elements in constant electron temperature plasmas, we estimated the iron ionization time scales to be several minutes for this particular flare (see bottom panel of Figure 4). Therefore, the $131 \AA$ signal associated with the RHESSI thermal emission is expected to have been delayed or even suppressed.

We also searched for signs of the $\mathrm{Fe}$ line complex around $6.7 \mathrm{keV}$ in the RHESSI data. Although the count spectra suggest that there might be a faint Fe line feature present, spectral fitting is inconclusive and does not give a quantitative result as it is unclear how to subtract the on-disk emission (see Section 3.1), making fitting the Fe line even more difficult than it already is for data taken late in the RHESSI mission lifetime when significant radiation damage to the detectors has been accumulated (these observations were taken 14 months past the last RHESSI anneal in February 2012).

Because the source was expanding while the density 


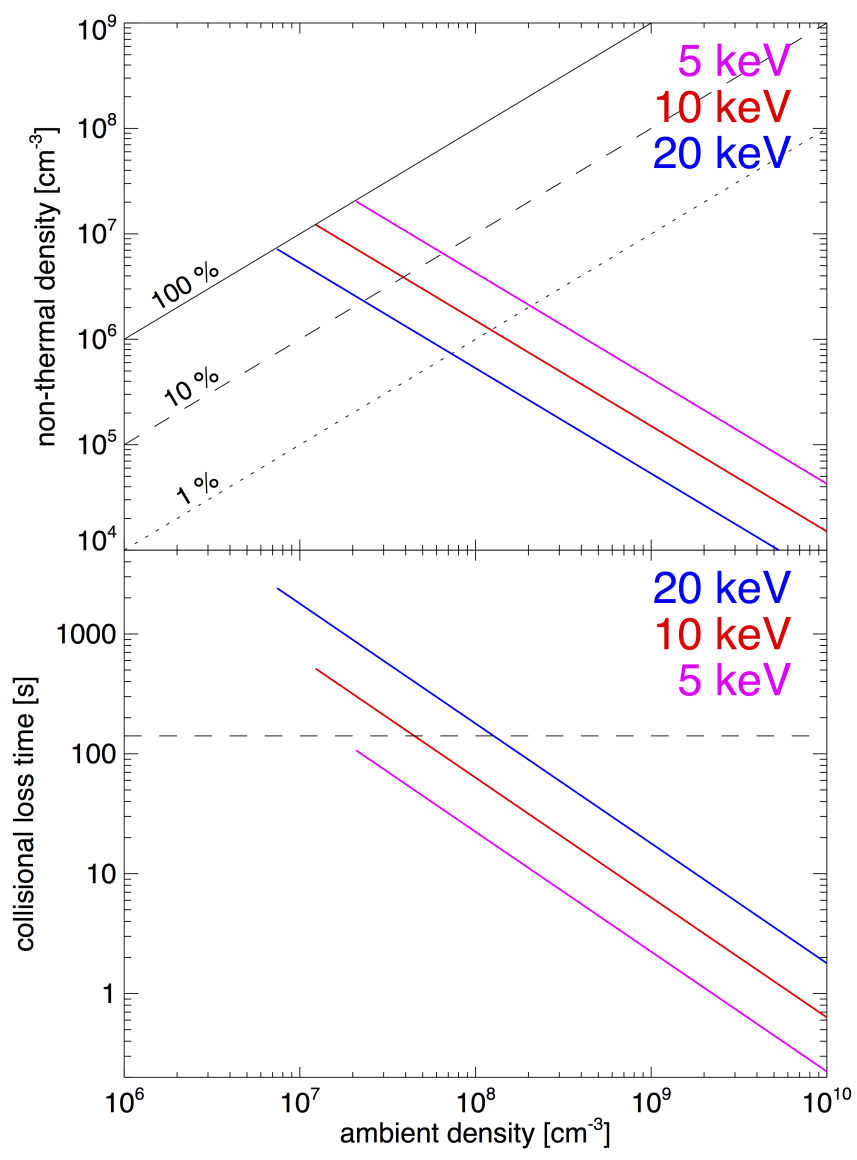

Figure 8. Non-thermal electron density (top) and collisional loss timescales (bottom) calculated for bremsstrahlung emission at different energies.

was decreasing, it is not straight forward to predict the actual flux that would have been observed in AIA $131 \AA$.

We nevertheless made a simple calculation to determine the hypothetical EUV intensity, assuming instantaneous ionization. Given the size and temperature of the soft X-ray source, we should see $550 \mathrm{~K}$ data numbers (DN) per second over the area of the source in $131 \AA$. In $94 \AA$ this number is much less, at only $41 \mathrm{~K} \mathrm{DN} / \mathrm{s}$. With the area calculated from the X-ray imaging, both channels should show a source whose intensity is easily observable above the background, once the plasma has become sufficiently ionized. Figure 9 shows what we could expect versus what was observed. The minimum ionization time of four minutes was on the order of the decay time of the RHESSI thermal emission. Other factors, such as the presence of flows or collisions with non-thermal particles might affect the time it takes for ionization equilibrium to be reached. However, because there are no signs of the plasma in AIA, this means that whatever the rate, the ionization simply could not catch up, regardless of the presence of non-equilibrium effects.

We also calculated the expected $17 \mathrm{GHz}$ gyrosynchrotron flux from the size, spectral index, and density of the non-thermal HXR source, using formula 2.16 from White et al. (2011). Only 1-10 sfu would be produced; From the temperature and emission measure calculated from the thermal X-ray source, the potential $17 \mathrm{GHz}$ emissions would be even less; using equation 15 from Morgachev et al. (2014), the contribution from the freefree emission would be only $0.035 \mathrm{sfu}$. Imaging at $17 \mathrm{GHz}$ using data from the Nobeyama Radioheliograph (NoRH) showed much stronger emissions. Ten-second cadence images, made at both 17 and $34 \mathrm{GHz}(17 \mathrm{GHz}$ is shown in the bottom-right panel of Figure 1), showed a bubble of cold material in thermal emission emerging over the limb and rapidly expanding. This cold material showed fluxes of up to $1000 \mathrm{sfu}$, bright enough to eclipse any emission from the same electron population producing the HXR source.

When the observed radio contours were overlaid on those from AIA's 50,000 K $304 \AA$ channel, the correlation immediately suggested that the majority of the emission seen in the radio was in fact due to thermal radiation. Coronal sources observed at $\mathrm{GHz}$ wavelengths have been interpreted as bubbles filled with nonthermal electrons (c.f. White et al. 2011); however this does not agree with the majority of the thermal emission observed in the radio for this event.

\section{DISCUSSION}

The earliest report of a purely coronal hard X-ray burst with a very hard spectrum reaching up to hundreds of $\mathrm{keV}$ was the famous event of March 30, 1969 (Frost and Dennis 1971). Although not directly mentioned in that paper, the flare was occulted (Badillo and Salcedo 1969). Using stereoscopic spectral observations of the February 16, 1984 flare, Kane et al. (1992) were the first to determine that HXR sources must be extended, greater than 100 " in size. The first observations with imaging were provided by Yohkoh HXT (Hudson et al. 2001), which confirmed that the coronal source was extended and furthermore, moving away from the Sun. The best imaging so far was done by Krucker et al. (2007) using RHESSI, which clearly showed large sources that expanded while moving away from the Sun. Eventually the sources inflated to sizes so large that even the smallest Fourier component observable by RHESSI did not have a significant signal. Glesener et al. (2013) observed a related though barely-occulted event which demonstrated that HXR-producing electrons could heat the CME core to temperatures around $10 \mathrm{MK}$. Table 2 summarizes the key observables for previously published events.

While it is not clear that all studies indeed describe the same type of event, they share several common characteristics:

- The hard X-ray profiles are rather simple with a broad peak followed by an exponential decay.

- The non-thermal part of the spectrum is generally hard and tends to further harden during the decay.

- Sources are spatially extended and move away from the Sun while expanding.

- Sources are observed behind the front of the escaping CME.

These facts support the current best explanation that these HXR sources are produced by bremsstrahlung emission from flare-accelerated electrons, which escape upward from the coronal acceleration region. Flareaccelerated electrons cannot escape freely from the Sun 

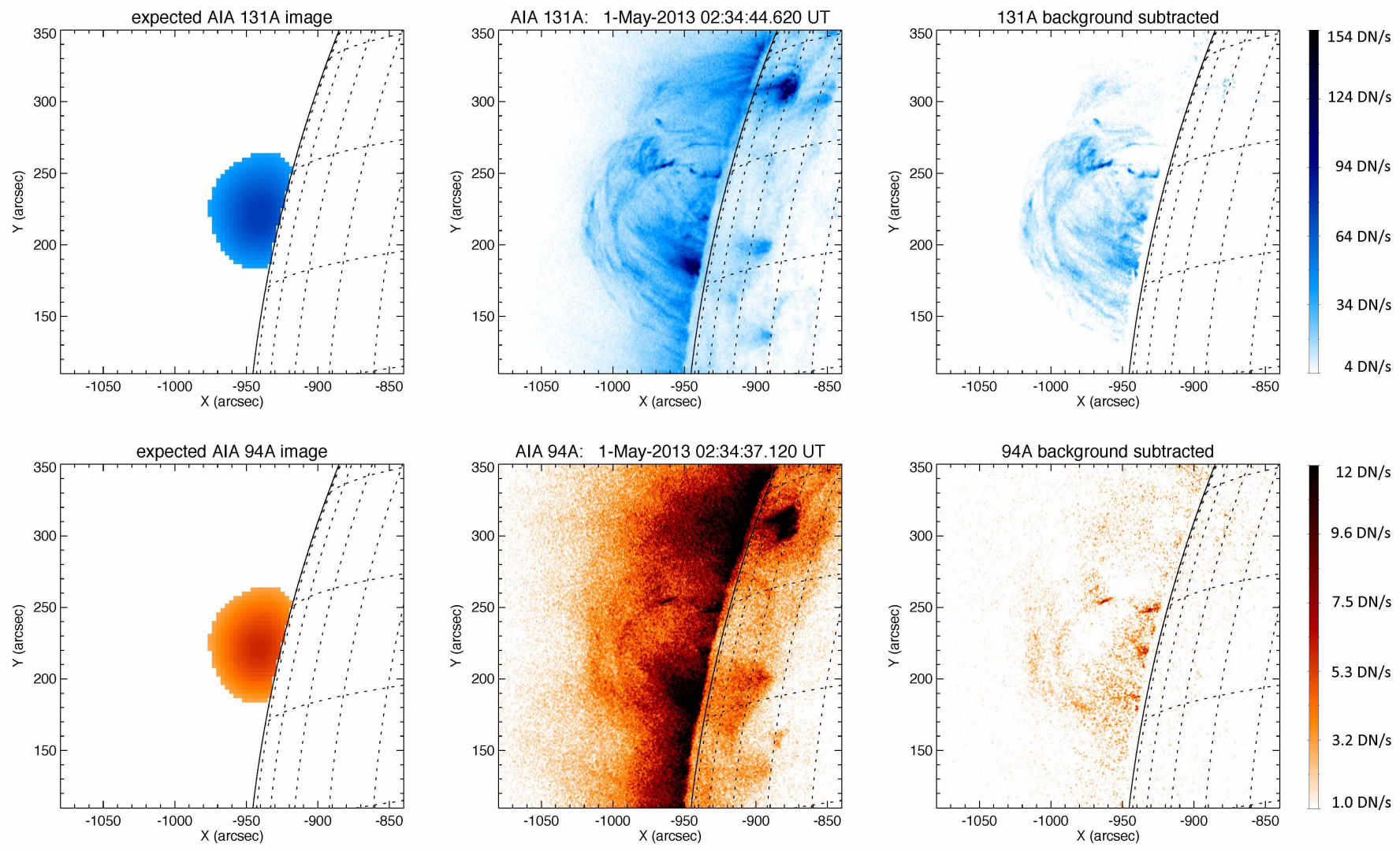

Figure 9. Left: Brightness of expected sources in AIA 131 and $94 \AA$, given the size, location, and electron density of the thermal X-ray source. Middle: AIA observations. Right: Pre-flare background-subtracted observed emission.

Table 1

Published properties of selected occulted flares

\begin{tabular}{|c|c|c|c|c|c|c|c|c|c|c|c|}
\hline Date & Publication & Occultation & $\begin{array}{l}\text { GOES } \\
\text { obs. } \\
\text { est. }\end{array}$ & $\begin{array}{l}\text { CME } \\
\text { speed } \\
(\mathrm{km} / \mathrm{s})\end{array}$ & $\begin{array}{l}30 \mathrm{keV} \\
\text { photon } \\
\text { flux }\end{array}$ & $\tau(\mathrm{s})$ & $\gamma_{c o r}$ & $\gamma_{\text {chro }}$ & $\begin{array}{l}30 \mathrm{keV} \\
\text { flux flare }\end{array}$ & $\begin{array}{c}\text { Size } \\
\text { arcsec }\end{array}$ & $\begin{array}{l}\text { Speed } \\
(\mathrm{km} / \mathrm{s})\end{array}$ \\
\hline $\begin{array}{l}\text { Mar 30, } \\
1969\end{array}$ & 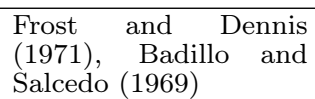 & $15^{\circ}$ & & & $\sim 3$ & $\sim 300$ & $\sim 2$ & & & & \\
\hline $\begin{array}{l}\text { Dec 14, } \\
1971\end{array}$ & Hudson (1978) & $25^{\circ}$ & & & 0.05 & $\sim 600$ & 2.1 & & & & \\
\hline $\begin{array}{l}\text { Jul 22, } \\
1972\end{array}$ & Hudson et al. 1982 & $20^{\circ}$ & & & 0.1 & $\sim 400$ & $2.5-1.8$ & & & & \\
\hline $\begin{array}{l}\text { Feb } 16, \\
1984\end{array}$ & Kane et al. (1992) & $36^{\circ}$ & B3 & & 0.4 & $\sim 60$ & $3.8-2.6$ & 3.3 & 150 & $140^{\circ}$ & \\
\hline $\begin{array}{l}\text { Jun } 30, \\
1991\end{array}$ & Vilmer et al. (1991) & $2 \pm 12^{\circ}$ & M5 & & 10 & $\sim 25$ & $2.8-2$ & & & & \\
\hline $\begin{array}{l}\text { Apr 18, } \\
2001\end{array}$ & Hudson et al. (2001) & $27^{\circ}$ & $\begin{array}{l}\mathrm{C} 2 \\
>\mathrm{X} 1\end{array}$ & 2400 & & $\sim 30$ & $4.3-3.4$ & & & $20 "-70 "$ & $\sim 1000$ \\
\hline $\begin{array}{l}\text { Oct } 27, \\
2003\end{array}$ & \begin{tabular}{|l|} 
Krucker et al. \\
Vybornov et al. \\
$\left(\frac{2007}{2012}\right)$
\end{tabular} & $40^{\circ}$ & $\begin{array}{l}\sim \mathrm{B} 1 \\
>\mathrm{X} 1\end{array}$ & 2300 & 0.1 & $\sim 135$ & $3.6-3.1$ & 2.3 & 80 & $200 "$ & $\sim 750$ \\
\hline $\begin{array}{l}\text { Nov } 3, \\
2012\end{array}$ & Glesener et al. 2013) & $6^{\circ}$ & $\begin{array}{l}\mathrm{C} 5 \\
\mathrm{X} 1\end{array}$ & 240 & 0.3 & $\sim 30$ & 4.5 & & & $50 "-100 "$ & \\
\hline $\begin{array}{l}\text { May } 1, \\
2013\end{array}$ & Lastufka et al. (2019) & $30^{\circ}$ & $\begin{array}{l}\text { B } \\
\text { M3-7 }\end{array}$ & 400 & 0.03 & $\sim 140$ & 3.3 & 2.5 & 20 & $100 "$ & $\sim 200$ \\
\hline $\begin{array}{l}\text { Sep } 1, \\
2014\end{array}$ & \begin{tabular}{|l|l} 
Carley et al. \\
Grechnev et al.
\end{tabular} & $36^{\circ}$ & $\begin{array}{l}\sim \mathrm{B} 1 \\
\mathrm{X} 2\end{array}$ & 2000 & 0.2 & $\sim 900$ & $2.06^{a}$ & 3.3 & 220 & $200 "$ & \\
\hline
\end{tabular}

${ }^{a}$ This spectral index was derived from flux integrated over the entire flare (Ackermann et al. 2017), whereas $\gamma_{\text {chro }}$ and the $30 \mathrm{keV}$ flux quoted are for the first X-ray peak seen by HEND 
because they are injected within the complex magnetic field structure of the CME core. Due to the low ambient density in the corona, these electrons only lose energy slowly; however, they can move around within the CME core during their livetime, resulting in a large source size.

The observed exponential decay is likely the result of the combined loss mechanisms after the injection stops. The largest contributor is probably Coulomb collisions, but electrons leaving the source volume can also result in a reduction of flux. Other loss mechanisms might be at play as well. It is also possible that acceleration might not stop completely after the event peak, such that continuing injection at a low rate prolongs the decay. Using estimates of the ambient density, the expected collisional loss times roughly agree with the observed time scales of the decay, indicating that collisions alone could be enough to explain the decaying time profile. The collisional losses are expected to heat the ambient plasma, creating a hot thermal source within the CME core (Glesener et al. 2013).

The observations of the May 1 flare discussed in this work fit well within this picture of large source sizes and exponential decay. What is different from the event in Glesener et al. (2013) is that the source of the nonthermal emission was spatially displaced from the hot CME core. Therefore, further evidence is required to conclude that the May $1 \mathrm{CME}$ core was heated by nonthermal electrons. If such heating did occur, it must have been from a HXR source co-spatial with the core that was no longer observable by the time the core became visible above the solar limb. This is certainly possible; comparing with the event studied by Glesener et al. (2013), the CME core would have been barely visible above the limb at the end time of the HXR burst (c.f. Figure 3, panel 6, from Glesener et al. (2013)) if it had had the same occultation height seen here for the May 1 event. However, this implies that the RHESSI non-thermal emission shown in Figure 7 must have come from a different population of energetic electrons than that of the observed HXR source. These could have been injected into a magnetic structure with a lower density, allowing the electrons to survive longer, or a second injection could have occurred at a later time. Nevertheless, we have no observational evidence for such a scenario so the prudent explanation is that that hot core in the May 1 flare was not heated by non-thermal particles, but rather a different mechanism.

For four events in Table 2, including the May 1, spectral observations of the main flare emission allow us to estimate the relative intensity of the coronal source with respect to the rest of the flare. Spectral information for full-flare perspective was only available above 100 $\mathrm{keV}$. High coronal events are best seen at lower energies around $30 \mathrm{keV}$, making an extrapolation necessary. Comparing the extrapolated fluxes at $30 \mathrm{keV}$, the total flare emission is 400 to 800 times stronger than the coronal emission. The flare spectra also tend to be harder in the chromosphere/low corona than in the high coronal source; hence, the difference in flux is expected to increase at higher energies with values between 1000 and 1500 at $100 \mathrm{keV}$.

While these flux ratios are relevant for detection, the physical significance comes from the number of accelerated electrons within each source. To derive the number
Table 2

Estimates of the number of electrons above $20 \mathrm{keV}$ assuming thick target emissions from the footpoints and thin target for the extended coronal source.

\begin{tabular}{lcc}
\hline Date & $\begin{array}{c}\text { Thick target } N_{e} \\
\text { footpoints } \\
>20 \mathrm{keV}\end{array}$ & $\begin{array}{c}\text { Thin target } N_{e} \\
\text { corona } \\
>20 \mathrm{keV} \text { at peak }\end{array}$ \\
\hline Feb 16, 1984 & $2.5 \times 10^{39}$ & $2.5 \times 10^{36}$ \\
Oct 27, 2003 & $3.5 \times 10^{38}$ & $6.7 \times 10^{35}$ \\
May 1, 2013 & $9.4 \times 10^{37}$ & $2.0 \times 10^{35}$ \\
Sept 1, 2014 & $2.7 \times 10^{39}$ & $7.7 \times 10^{35}$ \\
\hline
\end{tabular}

of electrons, we have to adopt a certain model. The total flare energy is usually derived by assuming that electrons lose all their energy in the chromosphere, with collisions being the main loss mechanism (e.g. cold thick target model, e.g. Brown (1971)). This is a rather robust assumption for chromospheric sources, but it depends on low-energy cutoff that is poorly constrained. We summarize the energy input to the chromosphere by electrons using $20 \mathrm{keV}$ as a reference in Table 3. While the coronal high-energy source is also likely produced by bremsstrahlung (for a discussion on the possible contribution of inverse Compton radiation see Chen and Bas$\operatorname{tian}(2012))$, the low ambient density within the source makes the classic thick target assumption inappropriate. Therefore the number of electrons needed to produce the hard X-ray spectrum at any time can be estimated from the instantaneous number of electrons by assuming a thin target model.

However, the instantaneous number $N_{e}$ depends not only on the observed photon spectrum (as does the thick target approximation), but also on the ambient density, to which it is inversely proportional. Using an ambient density of $10^{8} \mathrm{~cm}^{-3}$ for all events, Table 3 gives the instantaneous number of electrons at peak time, again with $20 \mathrm{keV}$ as the reference energy. $N_{e}$ at peak time is the maximum number of accelerated electrons that radiates at any time. It can therefore be used as a lower limit of the total electrons in the high-altitude source. The actual number could be larger, as a lower-magnitude injection of electrons could continue after the peak time. Particleacceleration occurring in flares well after the peak is a well-established behavior. Additionally, a larger number of electrons might have been injected before the nonthermal source became visible above the limb as seen from Earth. Considering all these uncertainties, and that the ambient density is not well constrained, the estimate of the number of electrons is uncertain by a factor of a few at least. Nevertheless, it is our current best estimate that compared to the number of $>20 \mathrm{keV}$ electrons in the main flare peak, the number of electrons in the high coronal source are below the percent range.

\section{CONCLUSION}

The moderately sized flare and CME of May 1, 2013 was uniquely well-situated for high-energy observations associated with CMEs. Due to the event's position $30^{\circ}$ behind the solar limb, RHESSI saw coronal emission with no contamination from the footpoints or flare loop arcade. From the opposite side of the Sun, HEND viewed the full, un-occulted flare. Radio and EUV instruments were available to provide context and constrain the interpretation of the event.

Analysis of the thermal X-ray emissions found that 
plasma with temperatures up to $11 \mathrm{MK}$ was ejected. The hot source was located behind and rose slower than the CME front, indicating that it was likely the result of hot plasma trapped in the complex magnetic fields of the CME core. RHESSI imaging of this large thermal X-ray source expanding as it rose above the flare site may be the best such observation to date. The long iron ionization time scales for such a high altitude source at low density made the hot core of the CME undetectable in EUV, unlike in Glesener et al. (2013).

Imaging also showed a clear short-lived non-thermal source, which was very extended with a FWHM of 110", located $185^{\prime \prime}$ in projection above the flaring region. This was both higher up than the thermal CME core, and still behind the CME front. It must have originated from a more tenuous part of the CME core where non-thermal electrons survived long enough to become visible from Earth. Because of their location above the CME core, the non-thermal particles in this source could not have been responsible for heating the core itself. It is possible that a different population of non-thermal electrons heated the core to its $11 \mathrm{MK}$ temperature, but these were not observed due to the occultation. Another possibility is that a different heating mechanism entirely was responsible. Either way, we were unable to say for certain how the CME core was heated.

Assuming a thick-target model for the HEND spectrum and a thin-target model for that of RHESSI, we deduced that the number of coronal vs chromospheric nonthermal electrons to be one in five hundred. Although some bursts were observed in the flaring region before the main peak, both RHESSI and HEND observed the main electron acceleration at almost the same time. HEND, whose count spectrum was dominated by the chromospheric footpoints, saw a thousand times more photon flux than RHESSI. Both this flux ratio and the spectral index difference agrees with earlier studies by Kane et al. (1992); Vybornov et al. (2012); however, the flares examined in these works were extremely large. With $\mathrm{X}$-ray imaging spectrometers, it is understandable that only the largest such events are identified, since larger flares produce more high-energy counts which improves the possibility and quality of imaging. The presence of similar properties in the M-class flare of May 1 invites us to consider whether such a flux ratio and spectral index difference are common to all flares regardless of magnitude. Furthermore, the presence of coronal X-ray sources very high above the site of an average-sized flare supports the idea that these should be present in most flares, and could be revealed given sufficient observations from various angles, as will be provided by Solar Orbiter.

\section{ACKNOWLEDGEMENTS}

The LASCO CME catalog is generated and maintained at the CDAW Data Center by NASA and The Catholic University of America in cooperation with the Naval Research Laboratory. SOHO is a project of international cooperation between ESA and NASA.

The work was supported by Swiss National Science Foundation (200021-163377) and through NASA contract NAS 5-98033 for RHESSI. The authors would like to thank the anonymous reviewer, whose comments im- proved the paper.

\section{REFERENCES}

M. Ackermann, A. Allafort, L. Baldini, G. Barbiellini, D. Bastieri, R. Bellazzini, E. Bissaldi, R. Bonino, E. Bottacini, J. Bregeon, P. Bruel, R. Buehler, R. A. Cameron, M. Caragiulo, P. A. Caraveo, E. Cavazzuti, C. Cecchi, E. Charles, S. Ciprini, F. Costanza, S. Cutini, F. D'Ammando, F. de Palma, R. Desiante, S. W. Digel, N. Di Lalla, M. Di Mauro, L. Di Venere, P. S. Drell, C. Favuzzi, Y. Fukazawa, P. Fusco, F. Gargano, N. Giglietto, F. Giordano, M. Giroletti, I. A. Grenier, L. Guillemot, S. Guiriec, T. Jogler, G. Jóhannesson, L. Kashapova, S. Krucker, M. Kuss, G. La Mura, S. Larsson, L. Latronico, J. Li, W. Liu, F. Longo, F. Loparco, P. Lubrano, J. D. Magill, S. Maldera, A. Manfreda, M. N. Mazziotta, W. Mitthumsiri, T. Mizuno, M. E. Monzani, A. Morselli, I. V. Moskalenko, M. Negro, E. Nuss, T. Ohsugi, N. Omodei, E. Orlando, V. Pal'shin, D. Paneque, J. S. Perkins,

M. Pesce-Rollins, V. Petrosian, F. Piron, G. Principe, S. Rainò, R. Rando, M. Razzano, O. Reimer, F. Rubio da Costa, C. Sgrò, D. Simone, E. J. Siskind, F. Spada, G. Spandre, P. Spinelli, H. Tajima, J. B. Thayer, D. F. Torres, E. Troja, and G. Vianello. Fermi-LAT Observations of High-energy Behind-the-limb Solar Flares. The Astrophysical Journal, 835: 219, February 2017. ISSN 0004-637X. doi: $10.3847 / 1538-4357 / 835 / 2 / 219$.

V. L. Badillo and J. E. Salcedo. Solar Microwave Burst from Behind-the-Limb Proton Flare. Nature, 224(5218):503, November 1969. ISSN 1476-4687. doi: 10.1038/224503a0.

M. Battaglia and A. O. Benz. Relations between concurrent hard X-ray sources in solar flares. $A \mathscr{G} A, 456(2): 751-760$, September 2006. ISSN 0004-6361, 1432-0746. doi:

10.1051/0004-6361:20065233.

Marina Battaglia, Hugh S. Hudson, Gordon J. Hurford, Säm Krucker, and Richard A. Schwartz. The Solar X-Ray Limb. ApJ, 843(2):123, July 2017. ISSN 0004-637X. doi: $10.3847 / 1538-4357 /$ aa76da.

W. V. Boynton, W. C. Feldman, I. G. Mitrofanov, L. G. Evans, R. C. Reedy, S. W. Squyres, R. Starr, J. I. Trombka, C. D'uston, J. R. Arnold, P. A. J. Englert, A. E. Metzger, H. Wänke, J. Brückner, D. M. Drake, C. Shinohara, C. Fellows, D. K. Hamara, K. Harshman, K. Kerry, C. Turner, M. Ward, H. Barthe, K. R. Fuller, S. A. Storms, G. W. Thornton, J. L. Longmire, M. L. Litvak, and A. K. Ton'chev. The Mars Odyssey Gamma-Ray Spectrometer Instrument Suite. In Christopher T. Russell, editor, 2001 Mars Odyssey, pages 37-83. Springer Netherlands, Dordrecht, 2004. ISBN 978-0-306-48600-5. doi: 10.1007/978-0-306-48600-5_2.

Stephen J. Bradshaw and John Raymond. Collisional and Radiative Processes in Optically Thin Plasmas. In André Balogh, Andrei Bykov, Peter Cargill, Richard Dendy, Thierry Dudok de Wit, and John Raymond, editors, Microphysics of Cosmic Plasmas, Space Sciences Series of ISSI, pages 195-230. Springer US, Boston, MA, 2014. ISBN 978-1-4899-7413-6.

John C. Brown. The deduction of energy spectra of non-thermal electrons in flares from the observed dynamic spectra of hard X-ray bursts. Sol Phys, 18(3):489-502, July 1971. ISSN 1573-093X. doi: 10.1007/BF00149070.

Eoin P. Carley, Nicole Vilmer, Paulo J. A. Simões, and Brían Ó Fearraigh. Estimation of a coronal mass ejection magnetic field strength using radio observations of gyrosynchrotron radiation. Astronomy and Astrophysics, 608:A137, December 2017. doi: 10.1051/0004-6361/201731368.

Bin Chen and T. S. Bastian. THE ROLE OF INVERSE COMPTON SCATTERING IN SOLAR CORONAL HARD $\mathrm{X}$-RAY AND $\$ \backslash$ upgamma $\$$-RAY SOURCES. ApJ, 750(1):35, April 2012. ISSN 0004-637X. doi: 10.1088/0004-637X/750/1/35.

Ilya Chertok, A.V. Belov, and V.V. Grechnev. A simple way to estimate the soft x-ray class of far-side solar flares observed with STEREO EUVI. Solar Physics, 290(7):1947-1961, May 2015. 
Brian R. Dennis and Rick L. Pernak. HARD X-RAY FLARE SOURCE SIZES MEASURED WITH THE RAMATY HIGH ENERGY SOLAR SPECTROSCOPIC IMAGER. The Astrophysical Journal, 698(2):2131-2143, June 2009. ISSN 0004-637X, 1538-4357. doi: 10.1088/0004-637X/698/2/2131.

K. J. Frost and B. R. Dennis. Evidence from Hard X-Rays for Two-Stage Particle Acceleration in a Solar Flare. The Astrophysical Journal, 165:655, May 1971. ISSN 0004-637X. doi: $10.1086 / 150932$.

Lindsay Glesener, Säm Krucker, Hazel M. Bain, and Robert P. Lin. Observation of Heating by Flare-accelerated Electrons in a Solar Coronal Mass Ejection. ApJL, 779(2):L29, 2013. ISSN 2041-8205. doi: 10.1088/2041-8205/779/2/L29.

V. V. Grechnev, V. I. Kiselev, L. K. Kashapova, A. A. Kochanov, I. V. Zimovets, A. M. Uralov, B. A. Nizamov, I. Y. Grigorieva, D. V. Golovin, M. L. Litvak, I. G. Mitrofanov, and A. B. Sanin. Radio, Hard X-Ray, and Gamma-Ray Emissions Associated with a Far-Side Solar Event. Sol Phys, 293(10):133, October 2018. ISSN 1573-093X. doi: 10.1007/s11207-018-1352-z.

R. A. Harrison. The nature of solar flares associated with coronal mass ejection. Astronomy and Astrophysics, 304:585, December 1995. ISSN 0004-6361.

R. A. Howard, J. D. Moses, A. Vourlidas, J. S. Newmark, D. G. Socker, S. P. Plunkett, C. M. Korendyke, J. W. Cook, A. Hurley, J. M. Davila, W. T. Thompson, O. C. St Cyr, E. Mentzell, K. Mehalick, J. R. Lemen, J. P. Wuelser, D. W. Duncan, T. D. Tarbell, C. J. Wolfson, A. Moore, R. A. Harrison, N. R. Waltham, J. Lang, C. J. Davis, C. J. Eyles, H. Mapson-Menard, G. M. Simnett, J. P. Halain, J. M. Defise, E. Mazy, P. Rochus, R. Mercier, M. F. Ravet, F. Delmotte, F. Auchere, J. P. Delaboudiniere, V. Bothmer, W. Deutsch, D. Wang, N. Rich, S. Cooper, V. Stephens, G. Maahs, R. Baugh, D. McMullin, and T. Carter. Sun Earth Connection Coronal and Heliospheric Investigation (SECCHI). Space Science Reviews, 136:67-115, April 2008. ISSN 0038-6308. doi: $10.1007 / \mathrm{s} 11214-008-9341-4$.

H. S. Hudson. A purely coronal hard X-ray event. The Astrophysical Journal, 224:235-240, August 1978. ISSN 0004-637X. doi: 10.1086/156370.

H. S. Hudson, R. P. Lin, and R. T. Stewart. Second-stage acceleration in a limb-occulted flare. Sol Phys, 75(1):245-261, January 1982. ISSN 1573-093X. doi: 10.1007/BF00153475.

H. S. Hudson, T. Kosugi, N. V. Nitta, and M. Shimojo. Hard X-Radiation from a Fast Coronal Ejection. The Astrophysical Journal, 561(2):L211, November 2001. doi: 10.1086/324760.

S. R. Kane, J. McTiernan, J. Loran, E. E. Fenimore, R. W. Klebesadel, and J. G. Laros. Stereoscopic observations of a solar flare hard X-ray source in the high corona. The Astrophysical Journal, 390:687-702, May 1992. ISSN 0004-637X. doi: 10.1086/171320.

Säm Krucker and R. P. Lin. Relative Timing and Spectra of Solar Flare Hard X-ray Sources. Solar Physics, 210(1):229, November 2002. doi: 10.1023/A:1022469902940.

Säm Krucker and R. P. Lin. Hard X-Ray Emissions from Partially Occulted Solar Flares. ApJ, 673(2):1181, 2008. ISSN 0004-637X. doi: 10.1086/524010.

Säm Krucker, S. M. White, and R. P. Lin. Solar Flare Hard $\mathrm{X}$-Ray Emission from the High Corona. The Astrophysical Journal Letters, 669:L49-L52, November 2007. ISSN 0004-637X. doi: $10.1086 / 523759$.

E. Landi, J. C. Raymond, M. P. Miralles, and H. Hara. Physical Conditions in a Coronal Mass Ejection from Hinode, Stereo, and SOHO Observations. ApJ, 711(1):75, 2010. ISSN 0004-637X. doi: 10.1088/0004-637X/711/1/75.
J.-Y. Lee, J. C. Raymond, Y.-K. Ko, and K.-S. Kim. Three-Dimensional Structure and Energy Balance of a Coronal Mass Ejection. ApJ, 692(2):1271, 2009. ISSN 0004-637X. doi: 10.1088/0004-637X/692/2/1271.

R. P. Lin. Non-relativistic solar electrons. Space Sci Rev, 16(1): 189-256, June 1974. ISSN 1572-9672. doi: $10.1007 / \mathrm{BF} 00240886$

Wei Liu, Vahé Petrosian, Brian R. Dennis, and Yan Wei Jiang. Double Coronal Hard and Soft X-Ray Source Observed by RHESSI : Evidence for Magnetic Reconnection and Particle Acceleration in Solar Flares. The Astrophysical Journal, 676 (1):704-716, March 2008. ISSN 0004-637X, 1538-4357. doi: 10.1086/527538.

MA Livshits, IV Zimovets, DV Golovin, BA Nizamov, VI Vybornov, IG Mitrofanov, AS Kozyrev, ML Litvak, AB Sanin, and VI Tretyakov. Catalog of hard x-ray solar flares detected with mars odyssey/hend from the mars orbit in 2001-2016. Astronomy Reports, 61(9):791-804, 2017.

S. Masuda, T. Kosugi, H. Hara, S. Tsuneta, and Y. Ogawara. A loop-top hard X-ray source in a compact solar flare as evidence for magnetic reconnection. Nature, 371(6497):495-497, October 1994. ISSN 1476-4687. doi: 10.1038/371495a0.

A. S. Morgachev, V. E. Polyakov, and V. F. Melnikov. Contribution of thermal bremsstrahlung to microwave emission of solar flare loops. Astronomy Reports, 58(5):335-344, May 2014. ISSN 1063-7729, 1562-6881. doi: $10.1134 / \mathrm{S} 1063772914040076$.

N. V. Nitta, M. J. Aschwanden, P. F. Boerner, S. L. Freeland, J. R. Lemen, and J.-P. Wuelser. Soft X-ray Fluxes of Major Flares Far Behind the Limb as Estimated Using STEREO EUV Images. Sol Phys, 288(1):241-254, November 2013. ISSN 0038-0938, 1573-093X. doi: 10.1007/s11207-013-0307-7.

Randall K. Smith and John P. Hughes. Ionization Equilibrium Timescales in Collisional Plasmas. The Astrophysical Journal, 718(1):583-585, July 2010. ISSN 0004-637X, 1538-4357. doi: 10.1088/0004-637X/718/1/583.

Nicole Vilmer, Gerard Trottet, Claude Barat, Richard A Schwartz, Shinzo Enome, Alexandr Kuznetsov, Rachid Sunyaev, and Oleg Terekhov. Hard X-ray and gamma-ray observations of an electron dominated event associated with an occulted solar flare. $A \& A, 342: 9,1991$.

V. I. Vybornov, M. A. Livshits, L. K. Kashapova, I. G. Mitrofanov, D. V. Golovin, A. S. Kozyrev, M. L. Litvak, A. B. Sanin, V. I. Tret'yakov, W. Boynton, K. Shinohara, and D. Hamara. Observation of the powerful solar flare of October 27, 2002 on the far side of the sun. Astronomy Reports, 56: 805-812, October 2012. ISSN 1063-7729. doi: 10.1134/S1063772912100083.

Stephen M. White, Arnold O. Benz, Steven Christe, Frantisek Farnik, Mukul R. Kundu, Gottfried Mann, Zongjun Ning, Jean-Pierre Raulin, Adriana V. R. Silva-Valio, Pascal Saint-Hilaire, Nicole Vilmer, and Alexander Warmuth. The Relationship Between Solar Radio and Hard X-ray Emission. Space Science Reviews, 159(1-4):225-261, September 2011. ISSN 0038-6308, 1572-9672. doi: 10.1007/s11214-010-9708-1.

J. Zhang, K. P. Dere, R. A. Howard, M. R. Kundu, and S. M. White. On the Temporal Relationship between Coronal Mass Ejections and Flares. ApJ, 559(1):452, 2001. ISSN 0004-637X. doi: $10.1086 / 322405$. 